\title{
Identificação de compostos orgânicos em lixiviado de aterro sanitário durante tratamento por processo fenton
}

Lixiviado de aterro sanitário é um dos subprodutos da decomposição biológica dos resíduos sólidos urbanos, sendo gerado no interior das células do aterro. Sua composição química está diretamente relacionada a idade a as características dos resíduos sólidos nele dispostos. Lixiviados mais velhos apresentam alto potencial poluidor, devido a presença de alta concentração de material orgânico de difícil biodegradação, macro poluentes inorgânicos, metais pesados e compostos orgânicos xenobióticos, sendo inviável o tratamento biológico. O presente trabalho teve como objetivo avaliar a eficiência do processo Fenton no tratamento de lixiviado proveniente do aterro sanitário do município de Puxinanã-PB, para isso foi realizado a caracterização química do Lixiviado "in natura" (LIN) e do Efluente Fenton (EF), e identificação de compostos orgânicos aplicando cromatografia gasosa acoplada a espectrometria de massa (CG-EM). Analisando os resultados podese constatar que o processo Fenton propiciou eficiência média de remoção de DQO total de $87 \%$, cor verdadeira de $98 \%$, COD de $74 \%$ e DBO5 de $70 \%$. A relação DBO5/DQO total no EF aumentou em um percentual de $100 \%$, quando comparado ao LIN. O processo Fenton removeu $80 \%$ dos compostos orgânicos identificados no LIN, dos 29 compostos que permaneceram no EF, alguns são reconhecidamente tóxicos como o cloro benzeno, bisfenol A, etil-benzeno e o 1-etil-2-metilBenzeno. Foi constatada, ainda, a formação de 50 novos compostos orgânicos como subprodutos da oxidação de compostos químicos no processo Fenton.

Palavras-chave: Lixiviado; Processo Fenton; Compostos orgânicos.

\section{Identification of organic compounds in leachate from landfill during treatment by fenton process}

\begin{abstract}
Leachate is one of the by-products of biological decomposition of solid urban waste, being generated inside landfills. Its composition is directly related to age and to the characteristics of the solid waste disposed in it. Older leachate have a high polluting potential, due to the high concentration of less degradable organic material, inorganic macro pollutants, heavy metals and organic xenobiotic compounds, making biological treatment is not viable. The present study aimed to evaluate the effectiveness of the Fenton process in the treatment of leachate from the landfill in Puxinanã-PB, for this purpose the characterization of leachate "in natura" (LIN) and effluent Fenton (EF) was conducted, physical-chemical parameters and identification of organic compounds using gas chromatography coupled with mass spectrometry (CG-EM). The results showed that the Fenton process provided an average removal efficiency of $87 \%$ total COD, $98 \%$ true color, $74 \%$ COD and $70 \%$ BOD5. The BOD5/total COD ratio of leachate treated by the Fenton process increased by a percentage of $100 \%$, when compared to LIN. The Fenton process removed $80 \%$ from the organic compounds identified in LIN, of the 29 compounds that remained in EF, some are known to be toxic with chlorine benzene, bisphenol A, ethyl benzene and 1-ethyl-2-methyl-benzene. It was also verified that 50 new organic compounds are formed as by-products of the oxidation of the Fenton process.
\end{abstract}

Keywords: Leachate; Fenton process; Organic compounds.

Topic: Engenharia Sanitária

Reviewed anonymously in the process of blind peer.
Received: 04/06/2021

Approved: 26/06/2021
Edilma Rodrigues Bento Dantas (iD)

Universidade Estadual da Paraíba, Brasil

http://lattes.cnpq.br/4120216485406294

http://orcid.org/0000-0001-9663-1787

edilma.dantas@hotmail.com

Wilza da Silva Lopes (iD)

Universidade Estadual da Paraíba, Brasil http://lattes.cnpq.br/7520507366596799 http://orcid.org/0000-0003-2391-6743

wilzasilvalopes@hotmail.com

José Tavares de Sousa

Universidade Estadual da Paraíba, Brasil

http://lattes.cnpq.br/9348418607084437

http://orcid.org/0000-0002-1056-1771

tavares@uepb.edu.br

\author{
Valderi Duarte Leite (in \\ Universidade Estadual da Paraíba, Brasil \\ http://lattes.cnpq.br/2319382787465258 \\ http://orcid.org/0000-0001-5861-7407 \\ mangabeiraleite@gmail.com

\section{Wilton Silva Lopes} \\ Universidade Estadual da Paraíba, Brasil \\ http://lattes.cnpq.br/1493216651945826 \\ http://orcid.org/0000-0002-0151-7664 \\ wiltonuepb@gmail.com
}

Referencing this:

DANTAS, E. R. B.; LOPES, W. S.; SOUSA, J. T.; LEITE, V. D.; LOPES, W. S.. Identificação de compostos orgânicos em lixiviado de aterro sanitário durante tratamento por processo fenton. Revista Ibero Americana de Ciências Ambientais, v.12, n.6, p.274-287, 2021. DOI: http://doi.org/10.6008/CBPC2179-6858.2021.006.0023 


\section{INTRODUÇÃO}

Lixiviado de aterro sanitário caracteriza-se por apresentar elevadas concentrações de matéria orgânica biodegradável e recalcitrante, de nitrogênio amoniacal, metais pesados, poluentes emergentes como as substâncias húmicas e fúlvicas (YUAN et al., 2016; SUN et al., 2015; RANI et al., 2020; CHRISTENSEN et al., 2001).

Cientes do desafio de tratar um resíduo líquido que apresenta composição química de alta complexidade como o lixiviado de aterro sanitário, países da União Europeia e outros países desenvolvidos como Estados Unidos, Japão e Dinamarca tem entendido que o aterro sanitário é a última opção na ordem de hierarquia na gestão dos resíduos sólidos urbanos, sendo enviado para ele apenas os rejeitos, o que aumentaria significantemente sua vida útil e diminuiria o volume de lixiviado produzido, evitando assim sua migração através de solos e subsolos diminuindo a possibilidade de contaminação de águas superficiais e subterrâneas (IYAMU et al., 2020; SAUVE et al., 2020; WANG et al., 2020; NJUKO et al., 2019; CHENG et at., 2020)

As tecnologias utilizadas no tratamento do lixiviado variam com base em vários fatores, dentre eles, os mais importantes são a composição química do lixiviado e idade do aterro (CHENG et al., 2020), processos físicos, químicos e biológicos podem ser utilizados, mas é importante salientar, que inevitavelmente o lixiviado de um mesmo aterro vai necessitar de diferentes tipos tecnologias ou tratamentos conjugados, visto que suas características mudam drasticamente com o passar do tempo (CASSANO et al., 2011; L-GOHARY et al., 2016; MIAO et al., 2019; AKKAYAA et al., 2020).

Lixividos jovens apresentam alta concentração de $\mathrm{DBO}_{5}$ e relação $\mathrm{DQO} / \mathrm{DBO}_{5}>0,5$, para estes, o tratamento biológico é o mais indicado, devido a simplicidade, confiabilidade e custo relativamente baixo (SALEEM et al., 2019; AKKAYA et al., 2020). Com o passar do tempo, a presença de compostos biodegradáveis vai diminuindo, prevalecendo compostos refratários (principalmente ácidos húmicos e fúlvicos) que são de difícil tratamento e por isso tendem a limitar a eficácia do processo biológico (RENOU et al., 2008; VILAR et al., 2011).

Lixiviados mais antigos são caracterizados por baixa concentração de $\mathrm{DBO}_{5}$, baixa relação $\mathrm{DQO} / \mathrm{DBO}_{5}$ $(<0,1)$, altas concentrações de nitrogênio amoniacal e de compostos orgânicos recalcitrantes (AKKAYA et al., 2020). Segundo Cassano et al. (2011), embora várias configurações de tratamento possam ser empregadas no tratamento de lixiviados antigos, a escolha do tratamento deve levar em consideração além das vantagens alcançadas, os custos e a eficácia do processo. Neste sentido, a utilização de processos oxidativos avançados $(\mathrm{POA})$, são interessantes, visto que, se baseiam na geração de radicais hidroxilas (HO•), espécie extremamente reativa e pouco seletiva, com potencial de oxidação de 2,8 V, que é capaz de degradar grande variedade de substâncias, inclusive compostos não biodegradáveis (UMAMAHESWARI et al., 2020; ANFRUNS et al., 2013; GAUTAM et al., 2019).

O processo Fenton foi descoberto em 1894 por Henry Fenton (FENTON, 1894) e é um dos processos oxidativos avançados (POAs) mais utilizados no tratamento de efluentes complexos como o lixiviado, e 
consiste na reação de íons de $\mathrm{Fe}^{2+}$ com peróxido de hidrogênio para formar espécies ativas $(\bullet \mathrm{OH})$ em meio ácido. Os radicais formados são capazes de degradar compostos orgânicos recalcitrantes e tóxicos (BABUPONNUSAMI et al., 2014). Segundo Göde et al. (2019) o processo Fenton pode ser resumidamente representado pelas equações Eqs. (1) e (2). Na Eq (1) são produzidos os radicais hidroxilas e na Eq (2) o catalisador $\mathrm{Fe}^{2+}$ é regenerado (BAUTISTA et al., 2008).

$$
\begin{array}{ll}
\mathrm{Fe}^{2+}+\mathrm{H}_{2} \mathrm{O}_{2} \rightarrow \mathrm{Fe}^{3+}+\mathrm{HO}^{-}+\mathrm{HO}^{-} & k=76 \mathrm{~L} \mathrm{~mol}^{-1} \mathrm{~s}^{-1} \\
\mathrm{Fe}^{3+}+\mathrm{H}_{2} \mathrm{O}_{2} \rightarrow \mathrm{Fe}^{2+}+\mathrm{HO}_{2}{ }^{-}+\mathrm{H}^{+} & \mathrm{k}=0,1 \mathrm{Lol}^{-1} \mathrm{~s}^{-1}
\end{array}
$$

Nogueira et al. (2007) advertem que $\mathrm{o}_{2} \mathrm{O}_{2}$ pode atuar como sequestrador de radical hidroxila (HO*), formando o radical hidroperoxila $\left(\mathrm{HO}_{2}{ }^{\circ}\right)$ que apresenta um menor potencial de redução que o radical hidroxila, prejudicando, portanto, o processo de degradação de compostos orgânicos. Para evitar a formação do radical $\mathrm{HO}_{2}{ }^{\bullet}$ é imprescindível que seja feito a otimização do processo para se garantir uma concentração satisfatório de radicais $\mathrm{HO}^{*}$. A otimização também é importante para evitar a formação de lodo químico, que é formado quando a oxidação dos constituintes do lixiviado não se realiza completamente, prevalecendo o processo de coagulação, nesta etapa ocorre a formação do lodo considerado um dos aspectos negativos do processo Fenton (MORAVIA et al., 2011; ISKANDER et al., 2019; WU et al., 2018).

Para aumentar a eficiência do processo Fenton e com isso reduzir a produção de lodo gerado, é extremamente importante que sejam feitos estudos preliminares visando a otimização do processo para cada efluente que se deseja tratar. Os parâmetros decisivos na boa condução do tratamento Fenton são, pH de oxidação e coagulação; concentração do ferro, e do peróxido de hidrogênio; razão molar $\left[\mathrm{H}_{2} \mathrm{O}_{2}\right] /\left[\mathrm{Fe}^{2+} \mathrm{e}\right.$ temperatura (DANTAS et al., 2019; KARTHIKEYAN et al., 2011; ZHANG et al., 2019; PLATA et al., 2010).

A eficácia do processo Fenton na remoção de material orgânico, nitrogenado e cor verdadeira já é bem documentada, mas são insuficientes, visto que vários compostos orgânicos das mais variadas classes podem contaminar solo, água e ar, deste modo, caracterizações mais robustas, que englobem parâmetros físicos, químicos e principalmente, identificação de compostos orgânicos podem auxiliar no entendimento do mecanismo de contribuição individual de cada composto orgânico e de suas interações na toxicidade do lixiviado (RIGOBELLO et al., 2015; PAIVA et al., 2011; REIS et al., 2017; MENDONÇA et al., 2009; HYBSKÁ et al., 2020; TIGINI et al., 2014).

Diante do exposto, é possível constatar que o aprofundamento na caracterização química de lixiviados de aterros sanitários é condição basilar, tanto na avaliação da eficácia dos tratamentos usualmente utilizados no Brasil, quanto para se obter um panorama dos principais contaminantes que estão presentes nos aterros brasileiros. Estes dados podem ser utilizados na predição da toxicidade do lixiviado para vários organismos de diferentes níveis tróficos e para se prever as interações dos compostos com o solo e com o ar. Por fim, tal caracterização pode sinalizar aos órgãos reguladores sobre a necessidade de mudança na legislação vigente, evidenciando a necessidade de monitoramento de compostos orgânicos potencialmente perigosos que são negligenciados nas atuais normas vigentes. Neste contexto, o objetivo deste estudo foi avaliar a eficiência do tratamento do lixiviado de aterro sanitário por processo Fenton através da caracterização química e da identificação de compostos orgânicos. 


\section{MATERIAIS E MÉTODOS}

\section{Amostragem e Otimização do Processo Fenton}

O Lixiviado utilizado na realização desta pesquisa foi coletado no aterro sanitário do município de Puxinanã-PB, que recebia em torno de 400 Toneladas/dia de resíduos sólidos urbano e gerava aproximadamente $4 \mathrm{~m}^{3} /$ dia de lixiviado.

Os parâmetros operacionais utilizados no processo Fenton foram otimizados por Dantas et al. (2019) e foram os seguintes, concentração de $\mathrm{Fe}^{2+}$ de $5 \mathrm{~g} / \mathrm{L}$, razão molar $\left[\mathrm{H}_{2} \mathrm{O}_{2}\right] /\left[\mathrm{Fe}^{2+}\right]$ de 9 , pH de oxidação 2 e pH de coagulação 8 , os tempos de oxidação, floculação e sedimentoção foram de 20, 10 e 15 min respectivamente.

Os ensaios foram realizados em escala de bancada, utilizando-se um equipamento Jar Teste marca Poli Control. Foram realizados 6 ensaios simultâneos com $1000 \mathrm{~mL}$ de lixiviado em cada jarra. Os íons Fe ${ }^{2+}$ foram obtidos do sulfato ferroso $\left(\mathrm{FeSO}_{4} .7 \mathrm{H}_{2} \mathrm{O}\right.$ - PA) na sua forma granular.

\section{Caracterização dos Parâmetros Físico e Químicos}

A determinação dos parâmetros físico-químicos foi realizada de acordo com os procedimentos recomendados pelo Standard Methods for the Examination of Water and Wastewater (APHA et al., 2012). Foram quantificados os seguintes parâmetros: demanda química de oxigênio (DQO), demanda bioquímica de oxigênio $\left(\mathrm{DBO}_{5}\right), \mathrm{pH}$, cor verdadeira, fósforo total, nitrogênio amoniacal $\left(\mathrm{N}-\mathrm{NH}_{4}{ }^{+}\right)$, nitrogênio total Kjeldah (NTK), e frações de sólidos. Carboidratos (DUBOIS et al., 1956), proteínas (FLOLUND et al., 1995), ferro

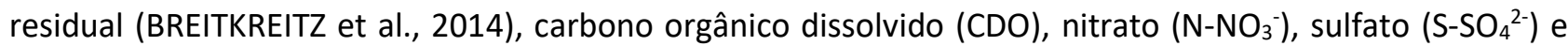
peróxido residual (NOGUEIRA et al., 2005).

\section{Caracterização qualitativa dos compostos orgânicos no LIN e no EF}

A extração dos compostos orgânicos foi realizada pelo método de Extração líquido-líquido (ELL) que está fundamentada na distribuição ou partição de um composto entre dois líquidos, nos quais este composto apresenta diferentes solubilidades (BEGUM et al., 2020). A identificação dos compostos orgânicos foi realizada por Cromatografia Gassosa acoplada a Espectro de Massa (CG-EM). A metodologia foi adaptada aos estudos previamente realizados para identificação de compostos orgânicos em lixiviado de aterro sanitário (RIGOBELLO et al., 2015; REIS et al., 2017).

Para o processo de ELL, uma amostra de lixiviado com pH ajustado para 2,0; 7,0 e 12 foi filtrada em membranas de acetato de celulose com poros de 0,45 $\mu \mathrm{m}$. Em um funil de separação, $100 \mathrm{~mL}$ desta amostra foi extraída por três vezes consecutivas com alíquotas de $30 \mathrm{~mL}$ de acetato de etila PA. Em seguida, a amostra foi centrifugada a $2500 \mathrm{rpm}$ por $10 \mathrm{~min}$. 0 sobrenadante (fase orgânica) foi transferido para um béquer de vidro e o precipitado foi descartado.

Em seguida foi adicionado à amostra $20 \mathrm{~g}$ de sulfato de sódio anidro $\left(\mathrm{Na}_{2} \mathrm{SO}_{4}-\mathrm{PA}\right)$ previamente seco em estufa a $105^{\circ}$ por 4 horas e filtrada em membrana de acetato de celulose com poros de $0.45 \mu \mathrm{m}$, e 
transferida para outro béquer de vidro. $\mathrm{O} \mathrm{Na}_{2} \mathrm{SO}_{4}$ foi lavado com $15 \mathrm{~mL}$ de acetato de etila PA, filtrado e transferido para o béquer com o extrato e posteriormente foi deixada em repouso para a evaporação do solvente para o volume final de $3 \mathrm{ml}$ sendo posteriormente injetado no CG-EM.

O processo de separação e identificação dos compostos orgânicos foi realizado em cromatógrafo (Thermo Scientific, Trace 1300 GC) acoplado a um espectrômetro de massa (Single Quadrupole Mass Spectrometer) e a uma Trace GOLD TG-5SILMS, $30 \mathrm{~m} \times 0.25 \mathrm{~mm} \times 0.25 \mu \mathrm{m}$. A programação da temperatura do forno foi $40^{\circ} \mathrm{C}$ por $2 \mathrm{~min}, 10^{\circ} \mathrm{C}$ por minuto até $70^{\circ} \mathrm{C} \mathrm{e} 5^{\circ} \mathrm{C}$ por minuto até $250^{\circ} \mathrm{C}$. Como gás de arraste, foi utilizado o gás Hélio, com 99.99\% de pureza, com um fluxo constante de $1 \mathrm{~mL}$. $\mathrm{min}^{-1}$. O volume de amostra injetado foi de $1 \mu \mathrm{l}$, com modo de injeção splitless a $250^{\circ} \mathrm{C}$ por min. A separação em CG foi alcançada usando o modo fullscan, com alcance de varredura de $50-500 \mathrm{~m} / \mathrm{z}$.

Os compostos orgânicos foram qualitativamente identificados por comparação espectral com a biblioteca do Instituto Nacional de Padrões e Tecnologia (National Institute of Standards and Technology, NIST), os espetros de massa foram comparados com os compostos de referência da biblioteca NIST.

\section{RESULTADOS E DISCUSSÃO}

\section{Caracterização do LIN e do EF}

Na Tabela 1 são apresentados os dados advindos a caracterização química do LIN e do EF. Em ralação a caracterização química do lixiviado "in natura" proveniente do aterro sanitário do município de Puxinanã$\mathrm{PB}$, pode-se observar elevadas concentrações de material orgânico expressas em termos de $\mathrm{DQO}, \mathrm{DBO}_{5} \mathrm{e}$ COD, $17988 \mathrm{mg} . \mathrm{L}^{-1} ; 6012 \mathrm{mg} . \mathrm{L}^{-1}$ e $1221 \mathrm{mg} . \mathrm{L}^{-1}$, respectivamente. No entanto, embora as concentrações de material orgânico sejam elevadas, a relação $\mathrm{DBO}_{5} / \mathrm{DQO}$ é moderada $(0,3)$, indicando biodegradabilidade relativamente baixa, tal fato, sugere a presença de compostos recalcitrantes no LIN. Um parâmetro importante para o entendimento do processo de degradação dos resíduos no interior dos aterros é o pH, pois o mesmo varia com o passar do tempo. $\mathrm{O}$ valor do $\mathrm{pH}$ do LIN era 8,8, esse dado corrobora com a baixa relação $\mathrm{DBO}_{5} / \mathrm{DQO}(0.3)$ visto que, quando o processo de degradação biológica da matéria orgânica se intensifica, os valores de $\mathrm{pH}$ vão se elevando em função do consumo dos ácidos pelas bactérias metanogênicas e pela maior produção de gases e neste estágio, a maioria das substâncias orgânicas biodegradáveis já foram degradas enquanto substâncias orgânicas refratárias continuam em processo de bioestabilização (POHLAND et al., 1985; XIAOLI et al., 2013).

Tabela 1: Caracterização do lixiviado “in natura” (LIN) e do Efluente Fenton (EF).

\begin{tabular}{l|l|l|l}
\hline Parâmetros & $\mathrm{LIN}$ & $\mathrm{EF}$ & \% Remoção \\
\hline $\mathrm{pH}$ & 8,8 & 7,80 & - \\
\hline $\mathrm{DQO}_{\mathrm{T}}(\mathrm{mg} / \mathrm{L})$ & $17988,0 \pm 12,7$ & $2230,0 \pm 75,1$ & 87 \\
\hline $\mathrm{DBO}_{5}(\mathrm{mg} / \mathrm{L})$ & $6012 \pm 17,7$ & $1721,2 \pm 75,1$ & 71,3 \\
\hline Cor verdadeira (uH) & $4900 \pm 14,1$ & $424,2 \pm 47,1$ & 91,3 \\
\hline $\mathrm{COD}(\mathrm{mg} / \mathrm{L})$ & $1221 \pm 3,5$ & $312,0 \pm 16,7$ & 74,4 \\
\hline Carboidratos (mg/L) & $932 \pm 4,2$ & $50,5 \pm 16,1$ & 94,6 \\
\hline Proteínas (mg/L) & $7235 \pm 140$ & $401,7 \pm 18$ & 94,4 \\
\hline Fósforo total (mg/L) & $51,6 \pm 1,3$ & $0,8 \pm 0,1$ & 98,4 \\
\hline $\mathrm{ST}(\mathrm{mg} / \mathrm{L})$ & $34074 \pm 5,7$ & $29055 \pm 2093,5$ & 14,7 \\
\hline $\mathrm{SV}(\mathrm{mg} / \mathrm{L})$ & $10221 \pm 1,4$ & $2969 \pm 300,2$ & $70,9 / 0$ \\
\hline
\end{tabular}




\begin{tabular}{|c|c|c|c|}
\hline $\mathrm{SF}(\mathrm{mg} / \mathrm{L})$ & $23853 \pm 9,9$ & $26086 \pm 1767,9$ & - \\
\hline SDT (mg/L) & $31157 \pm 3,5$ & $29662 \pm 797,7$ & 4,8 \\
\hline $\mathrm{SDV}(\mathrm{mg} / \mathrm{L})$ & $9005 \pm 7,1$ & $3850 \pm 423,2$ & 57,2 \\
\hline $\mathrm{SDF}(\mathrm{mg} / \mathrm{L})$ & $22152 \pm 4,2$ & $25812 \pm 761,6$ & - \\
\hline $\mathrm{N}-\mathrm{NTK}(\mathrm{mg} / \mathrm{L})$ & $2155 \pm 7,1$ & $216,3 \pm 17$ & 90,0 \\
\hline $\mathrm{N}-\mathrm{NH}^{+}{ }_{4}$ amoniacal (mg/L) & $1955 \pm 77,8$ & $143,5 \pm 6,8$ & 85 \\
\hline Ferro Residual (mg/L) & $2,7 \pm 0,1$ & $1,5 \pm 0,1$ & 44,4 \\
\hline NitratoN-NO $\mathrm{NO}_{3}(\mathrm{mg} / \mathrm{L})$ & $3,5 \pm$ & $48,8 \pm 25,3$ & - \\
\hline Sulfato $\mathrm{S}^{-\mathrm{SO}_{4}}{ }^{2-}(\mathrm{mg} / \mathrm{L})$ & $1307 \pm$ & $22083,3 \pm 5371,8$ & - \\
\hline $\begin{array}{l}\text { Peróxido Residual (mg/L) } \\
\mathrm{mg} / \mathrm{L}\end{array}$ & - & $0,4 \pm 0,1$ & - \\
\hline Temperatura (으) & 26 & 25 & - \\
\hline
\end{tabular}

Os compostos orgânicos refratários presentes no lixiviado são constituídos principalmente de substâncias húmicas e fúlvicas (variando em proporção de aterro para aterro) e são formadas por processos químicos e biológicos durante a degradação das substâncias orgânicas (KANG et al., 2002; YUAN et al., 2016; SUN et al., 2015).

Outra informação importante é que, quanto mais velho o aterro, maior será o grau de humificação e quantidade de componentes refratários presentes no lixiviado, tal fato é preocupante visto que, devido a suas características estruturais, as substâncias húmicas podem interagir com metais e compostos orgânicos (pesticidas e herbicidas) presentes no ambiente, o que pode potencializar a toxicidade do lixiviado (HUO et al., 2008; ROSA et al., 2000).

Além da elevada concentração de material orgânico, o nitrogênio amoniacal é uma grande preocupação ambiental, pois é liberado dos resíduos principalmente pela decomposição das proteínas e dependendo da forma prevalecente $\left(\mathrm{NH}_{3} \mathrm{Ou} \mathrm{NH}{ }_{4}^{+}\right)$a concentração no lixiviado pode ser mais ou menos tóxica (FERNANDES et al., 2015). No LIN também foi encontrada alta concentração de nitrogênio amoniacal, 1955 mg. $\mathrm{L}^{-1}$, como o $\mathrm{pH}$ do lixiviado era 8,8 maior parte do nitrogênio está na forma ionizada $\left(\mathrm{NH}_{4}^{+}\right)$, que não é tóxica, mas a tendência é que o pH do lixiviado aumente (desde que não haja diluição) o que resultaria no aumento de toxicidade devido o deslocamento da reação no sentido de formação de $\mathrm{NH}_{3}$.

De acordo com Xu et al. (2018) lixiviados com as características acima retratadas, não podem ser tratados apenas por métodos biológicos, visto que as técnicas convencionais de tratamento são bastantes limitadas e ineficientes na remoção de poluentes refratários, desse modo, o processo Fenton é indicado como pré-tratamento de lixiviados com estas características (ROUDI et al., 2019).

Observando os dados deste trabalho, percebe-se que o processo Fenton alcançou uma boa eficiência na remoção de DQO e cor verdadeira de $87 \%$ e $91 \%$ respectivamente. Estes altos valores de remoção são característicos do processo Fenton, principalmente quando há otimização dos paramentos operacionais, especialmente concentração de $\mathrm{Fe}^{2+}$, $\mathrm{pH}$ de oxidação e razão molar $\mathrm{H}_{2} \mathrm{O}_{2} / \mathrm{Fe}^{2+}$, sendo este último um dos parâmetros mais importante na eficiência do tratamento (ROUDI et al., 2020; SABOUR et al. 2017).

Resultados semelhantes foram encontrados por Moravia et al. (2011); Méndez et al. (2010) e Liu et al. (2020) que alcançaram bons resultados na remoção de $\mathrm{DQO}, \mathrm{DBO}_{5}$ e nitrogênio amoniacal, no entanto, melhores resultados são alcançados quando o lixiviado tratado é submetido a outro tipo de tratamento além do processo Fenton. Liu et al. (2020) alcançou excelentes resultados no tratamento de lixiviado proveniente 
de um aterro da Virgínia EUA, quando associou processo Fenton com Reator Eletroquímico com Membrana (REM), o processo Fenton isoladamente removeu aproximadamente $76 \%$ de DQO, enquanto que o processo REM-Fenton removeu $88 \%$. O lodo químico gerado no processo Fenton foi mais de $50 \%$ superior do que o gerado no processo REM-Fenton, as quantidades do reagente Fenton $\left(\mathrm{FeSO}_{4} .7 \mathrm{H}_{2} \mathrm{O}\right.$ e $\left.\mathrm{H}_{2} \mathrm{O}_{2}\right)$ também foram menores no REM-Fenton. O melhor resultado atingido pelo MER-Fenton foi na remoção 98\%. MORAVIA et al. (2013) alcançou excelentes resultados associando o processo Fenton com microfiltração (MF) e nanofiltração (NF), as remoções de DQO foram de 62\% e 72\% respectivamente. Poblete et al. (2020) utilizaram filtração, seguido de adsorção com serragem como pré-tratamento de lixiviado do aterro de El Panul (Chile) e posteriormente utilizaram solar photo-Fenton e solar photo-Fenton $+\mathrm{O}_{3}$. $\mathrm{O}$ tratamento combinado mostrou uma remoção de poluentes muito elevada, com redução de 95\% de DQO, 95\% de cor, 94\% de amônio e $98 \%$ de ácido húmico.

Alta concentração de DQO remanescente $\left(2230 \mathrm{mg} \cdot \mathrm{L}^{-1}\right)$ deve estar relacionada possivelmente pela formação de produtos intermediários estáveis que requerem um maior tempo para oxidação, às substâncias húmicas também podem ser responsáveis uma vez que podem estar solubilizada no meio, uma vez que os ácidos húmicos e fúlvicos são solúveis em soluções alcalinas (MORAVIA et al., 2011; LANGE et al., 2006). O processo Fenton é capaz de oxidar vários compostos orgânicos das mais variadas funções como álcoois, aldeídos, aminas e compostos aromáticos, mas é ineficaz na oxidação de ácido acético, acetona, triclorometano, ácido maleico, ácido malônico, ácido oxálico, n-parafinas, tetraclorometano.

A remoção de sólidos totais dissolvidos foi muito baixa, inferior a $5 \%$. No entanto foi observada a diminuição dos sólidos dissolvidos voláteis o que mostra o poder de oxidação do processo Fenton. No EF a concentração de nitrogênio amoniacal foi de $143,5 \mathrm{mg} / \mathrm{L}$, ou seja, o processo Fenton alcançou uma remoção de $85 \%$ quando comparado com ao LIN.

Essa remoção de nitrogênio amoniacal foi devido ao aumento do pH do LIN durante a oxidação da matéria orgânica e a agitação do líquido na mesma etapa. $\mathrm{O}$ aumento do pH favorece o deslocamento da reação no sentido de formação de fração gasosa. A agitação do lixiviado propicia a dessorção que é um processo físico de remoção de amônia da fase liquida devido ao aumento da superfície de contato total da fase aquosa com as hastes de agitação do jas test.

\section{Extração e Identificação de Compostos Orgânicos}

No total foram identificados 233 compostos orgânicos, destes, 154 foram identificados apenas no LIN e 79 no EF. Por se tratar de uma matriz ambiental extremamente complexa (alta heterogeneidade) a similaridade dos compostos identificados foi bastante variada, na Tabela 2 são apresentados alguns dos compostos orgânicos identificados por CG-MS no lixiviado in natura e no efluente Fenton, todos com similaridade superior a $50 \%$.

Tabela 2: Compostos orgânicos identificados por CG-EM no lixiviado de aterro sanitário do município de Puxinanã- PB, antes e após o tratamento Fenton, com similaridade acima de $50 \%$.

\begin{tabular}{|c|c|c|c|}
\hline Composto Orgânico & Fórmula & LIN & EF \\
\hline Ácido pentadecanóico & $\mathrm{C}_{15} \mathrm{H}_{30} \mathrm{O}_{2}$ & $\mathrm{X}$ & \\
\hline
\end{tabular}




\begin{tabular}{|c|c|c|c|}
\hline 2,4,6-trimetil-ácido benzoico & $\mathrm{C}_{10} \mathrm{H}_{12} \mathrm{O}_{2}$ & $\mathrm{x}$ & \\
\hline Ácido-dodecanóico & $\mathrm{C}_{12} \mathrm{H}_{24} \mathrm{O}_{2}$ & $\mathrm{x}$ & \\
\hline 3-etil-5-metil-heptano & $\mathrm{C}_{10} \mathrm{H}_{22}$ & $\mathrm{x}$ & \\
\hline 1,3-bis(1-metil-etil)-benzeno & $\mathrm{C}_{12} \mathrm{H}_{14}$ & $\mathrm{X}$ & \\
\hline Hexametil-ciclo-tri-siloxano & $\mathrm{C}_{6} \mathrm{H}_{18} \mathrm{O}_{3} \mathrm{Si}_{3}$ & $\mathrm{x}$ & \\
\hline 1,3 isobenzo-furanodiona & $\mathrm{C}_{8} \mathrm{H}_{4} \mathrm{O}_{3}$ & $\mathrm{X}$ & \\
\hline 11-hydroxi-4-metóxy-5-metil-dioxolo[4,5b]acridin-10[5H]-ona & $\mathrm{C}_{16} \mathrm{H}_{13} \mathrm{NO}_{5}$ & $\mathrm{x}$ & \\
\hline 2-metil-butanol & $\mathrm{C}_{5} \mathrm{H}_{12} \mathrm{O}$ & $\mathrm{X}$ & \\
\hline 2-etilamino-1-fenil-propanol & $\mathrm{C}_{11} \mathrm{H}_{17} \mathrm{NO}$ & $\mathrm{X}$ & \\
\hline $\mathrm{N}, \mathrm{N}$-dimetil-2,2-dimetil-[(3,5 dimetóxi) fenil]-etanamina & $\mathrm{C}_{16} \mathrm{H}_{27} \mathrm{NO}_{2}$ & $\mathrm{X}$ & \\
\hline Metil-tetra-decanoato de 12-Propil & $\mathrm{C}_{18} \mathrm{H}_{36} \mathrm{O}_{2}$ & $\mathrm{X}$ & \\
\hline Octadecil-fenil-ester-ácido carbónico & $\mathrm{C}_{25} \mathrm{H}_{42} \mathrm{O}_{3}$ & $\mathrm{X}$ & \\
\hline Di-isooctil-ftalato & $\mathrm{C}_{24} \mathrm{H}_{38} \mathrm{O}_{4}$ & $\mathrm{x}$ & \\
\hline 2-metil-benzeno-sulfonamida & $\mathrm{C}_{7} \mathrm{H}_{9} \mathrm{NO}_{2} \mathrm{~S}$ & $\mathrm{X}$ & \\
\hline $\begin{array}{l}\text { N-etil-2-metil-benzeno-sulfonamida } \\
\text { N-butylbenzenesulfonamide } \\
\text { N-Ethyl-2-methylbenzenesulfonamide } \\
\text { N-ethyl-4-methylbenzenesulfonamide }\end{array}$ & $\mathrm{C}_{9} \mathrm{H}_{13} \mathrm{NO}_{2} \mathrm{~S}$ & $\mathrm{X}$ & \\
\hline 2-hidroxi-1-(hidroximetil)-etil ester ácido hexadecanóico & $\mathrm{C}_{19} \mathrm{H}_{38} \mathrm{O}_{4}$ & $\mathrm{X}$ & \\
\hline 1-(2,4,6-trimetil-fenil)-etilamina-n-óxido-benzidrolideno & $\mathrm{C}_{24} \mathrm{H}_{25} \mathrm{NO}$ & $\mathrm{x}$ & \\
\hline Metoxi-fenil-oxima & $\mathrm{C}_{8} \mathrm{H}_{9} \mathrm{NO}_{2}$ & $\mathrm{X}$ & \\
\hline 2,2,6,6-tetrametil-4-piperidina & $\mathrm{C}_{9} \mathrm{H}_{17} \mathrm{NO}$ & $\mathrm{X}$ & \\
\hline Fenantreno & $\mathrm{C}_{14} \mathrm{H}_{10}$ & $\mathrm{X}$ & \\
\hline 1-metil-sulfonil-butano & $\mathrm{C}_{5} \mathrm{H}_{12} \mathrm{O}_{2} \mathrm{~S}$ & $\mathrm{x}$ & \\
\hline a,a-dihidroxi-m-diisopropil-benzeno & $\mathrm{C}_{12} \mathrm{H}_{18} \mathrm{O}_{2}$ & $\mathrm{X}$ & \\
\hline Octatômico Ciclico enxofre & $\mathrm{S}_{8}$ & $\mathrm{X}$ & \\
\hline Fenol & $\mathrm{C}_{6} \mathrm{H}_{6} \mathrm{O}$ & $\mathrm{X}$ & \\
\hline 2-butoxi-etanol & $\mathrm{C}_{6} \mathrm{H}_{14} \mathrm{O}_{2}$ & $\mathrm{x}$ & $\mathrm{x}$ \\
\hline Ácido hexadecanóico & $\mathrm{C}_{16} \mathrm{H}_{32} \mathrm{O}_{2}$ & $\mathrm{x}$ & $\mathrm{X}$ \\
\hline 1,1-(1,2-dimetil-1,2-etanodiil) bis ciclo-hexano & $\mathrm{C}_{16} \mathrm{H}_{30}$ & $\mathrm{x}$ & $\mathrm{x}$ \\
\hline Benzaldeído & $\mathrm{C}_{5} \mathrm{H}_{12} \mathrm{O}_{4}$ & $\mathrm{x}$ & $\mathrm{x}$ \\
\hline Ácido benzoico & $\mathrm{C}_{7} \mathrm{H}_{6} \mathrm{O}_{2}$ & $\mathrm{X}$ & $\mathrm{X}$ \\
\hline Cloro-benzeno & $\mathrm{C}_{6} \mathrm{H}_{5} \mathrm{Cl}$ & $\mathrm{X}$ & $\mathrm{X}$ \\
\hline 1-etil-2-metil-Benzeno & $\mathrm{C}_{9} \mathrm{H}_{12}$ & $\mathrm{X}$ & $\mathrm{X}$ \\
\hline Etil-Benzeno & $\mathrm{C}_{8} \mathrm{H}_{10}$ & $\mathrm{X}$ & $\mathrm{X}$ \\
\hline 4,4-isopropilideno-di-fenol (Bisfenol A) & $\mathrm{C}_{15} \mathrm{H}_{16} \mathrm{O}_{2}$ & $\mathrm{x}$ & $\mathrm{x}$ \\
\hline 4-hidroxi-4-metil-2-pentanona & $\mathrm{C}_{6} \mathrm{H}_{12} \mathrm{O}_{2}$ & $\mathrm{x}$ & $\mathrm{X}$ \\
\hline Benzoato de butila & $\mathrm{C}_{11} \mathrm{H}_{14} \mathrm{O}_{2}$ & $\mathrm{x}$ & $\mathrm{x}$ \\
\hline Carbonato de difenil & $\mathrm{C}_{13} \mathrm{H}_{10} \mathrm{O}_{3}$ & $\mathrm{x}$ & $\mathrm{X}$ \\
\hline Ácido octadecanóico & $\mathrm{C}_{18} \mathrm{H}_{36} \mathrm{O}$ & $\mathrm{X}$ & $\mathrm{X}$ \\
\hline 1,3-dimetil-benzeno (m-xileno) & $\mathrm{C}_{8} \mathrm{H}_{10}$ & & $\mathrm{X}$ \\
\hline Naftaleno & $\mathrm{C}_{10} \mathrm{H}_{8}$ & & $\mathrm{X}$ \\
\hline 2,4-di-tercbutil-fenol & $\mathrm{C}_{14} \mathrm{H}_{22} \mathrm{O}$ & & $\mathrm{X}$ \\
\hline 7,9-Di-tercbutil-1-oxaspiro [4.5] deca-6,9-dieno-2,8-dione & $\mathrm{C}_{17} \mathrm{H}_{24} \mathrm{O}_{3}$ & & $\mathrm{x}$ \\
\hline 13-docosenamida & $\mathrm{C}_{22} \mathrm{H}_{43} \mathrm{NO}$ & & $\mathrm{X}$ \\
\hline
\end{tabular}

Lin: Lixiviado "in natura"; EF: Efluente Fenton; X: Presença dos compostos orgânicos.

Os compostos identificados pertencem as mais variadas classes, ácidos carboxílicos, hidrocarbonetos aromáticos, alifáticos e cíclicos; álcoois, amidas, aminas, cetonas, ésteres, ftalatos e vários outros compostos de funções mistas. No LIN, a maioria dos compostos identificados (51\%) pertencem às seguintes classes químicas, alcanos (15\%), ácidos carboxílicos (11\%), ésteres (11\%), hidrocarbonetos aromáticos (8\%) e álcoois (6\%).

Alguns dos compostos identificados merecem ser destacados por serem reconhecidamente classificados como tóxico, são eles, o Di-isoctil ftalato, o Di-(2-propilpentil) éster ftálico e o 4,4-isopropilidenodi-fenol mais conhecido com Bisfenol A.

Tais compostos químicos são classificados como desreguladores endócrinos e sua presença no meio ambiente é preocupante, uma vez que estas substâncias podem causar danos ambientais mesmo em 
concentrações muito baixas ( $\mu \mathrm{g} \mathrm{L}^{-1}$ ou $\mathrm{ng} \mathrm{L}^{-1}$ ) e são micropoluentes que podem causar efeitos sérios a saúde humana como câncer de mama, redução da fertilidade masculina e feminina, gravidez anormal e aumento da incidência de ovários policísticos, pois são substâncias exógenas ao corpo humano e que interferem na síntese, secreção, transporte, metabolismo ou eliminação das diferentes hormônios (BILA et al., 2007).

O bisfenol A é um composto utilizado na fabricação de resinas epóxi e policarbonatos e é comumente encontrado em lixiviados de aterros sanitários (XIANGLI et al., 2006). Este composto químico já foi identificado em alguns lixiviados de aterros brasileiros localizados em Gravataí-RS (NASCIMENTO FILHO et al., 2001), Maringá-PR (RIGOBELLO et al., 2015) e Sabará-MG (REIS et al., 2017). Ftalatos como o di-isooctil ftalato são geralmente encontrados em plásticos maleáveis como o cloreto de polivinil, cosméticos, produtos de higiene pessoal, tintas para impressão, adesivos, tintas para pintura e selantes (WEI et al., 2015).

Outro composto identificado no LIN foi o fenantreno, que é um hidrocarboneto aromático policíclico (PAHs) que constitui uma classe de poluentes orgânicos persistentes e pode surgir naturalmente ou antropogenicamente. Fontes comuns de PAHs incluem combustíveis fósseis, vulcões, motores de combustão ou produção industrial de asfalto e alcatrão de carvão (SIMÃO et al., 2020).

Em lixiviado de aterros sanitários normalmente poderá haver a presença de matéria orgânica aromática, que ao contrário dos PAHs, são monoaromáticos e apresentam prioritariamente de 4 a 12 carbonos na sua estrutura e são considerados extremamente recalcitrantes e são normalmente encontrados na gasolina e são considerados poderosos depressores de sistema nervoso central (SILVA et al., 2009). Foram identificados aproximadamente 20 compostos monoaromático no LIN do aterro sanitário do município de Puxinanã-PB. Além dos compostos monoaromáticos, foram identificados dois compostos fenólicos, o fenol e o 4-(2-propenil)-fenol. Estes compostos são classificados como contaminantes orgânicos persistentes, apresentando assim, baixa biodegradabilidade, podendo causar sérios risco ao meio ambiente (DU et al., 2006).

Outros compostos fenólicos comumentes encontrados em aterros sanitários brasileiros são o 4metil-fenol, 4-(2-hidroxietil)-fenol, 4-terc-butil-fenol, 2,4-bis-fenol e o 4-metil-fenol (RIGOBELLO et al., 2015; REIS et al., 2017; TURKI et al., 2013).

Além dos parâmetros químicos, o processo Fenton se mostrou eficiente na remoção de $80 \%$ dos compostos identificados no LIN, tal fato se justifica pela baixa seletividade dos radicais hidroxila que é capaz de degradar compostos orgânicos das mais variadas classes (PERALTA-ZAMORA et al., 1999; PELLENZ et al., 2020). Vários compostos orgânicos permaneceram no EF, dentre eles, o cloro benzeno, bisfenol $A$, etilbenzeno e o 1-etil-2-metil-Benzeno. Como o trabalho visou apenas a identificação qualitativa, não foi possível avaliar se houve redução da concentração dos compostos orgânicos remanescentes. No entanto, foi observado remoção completa de compostos da classe dos siloxanos, heterocíclicos aromáticos e éter como pode ser observado na Figura 1.

É possível observar ainda, a ocorrência de formação de vários subprodutos do processo Fenton nas mais variadas classes de compostos identificados, esse é um aspecto negativo já esperado nesse tipo de tratamento. No EF foi observada a formação de mais de 50 novos compostos químicos, alguns com 
características tóxicas mais acentuadas que as dos compostos que os originaram, como é o caso do naftaleno e o 2,4-di-tercbutil-fenol e o naftaleno. Embora o processo Fenton esteja associado a alta eficiência na remoção dos compostos orgânicos, a formação de subprodutos é uma possibilidade real e isto gera limitações neste processo.

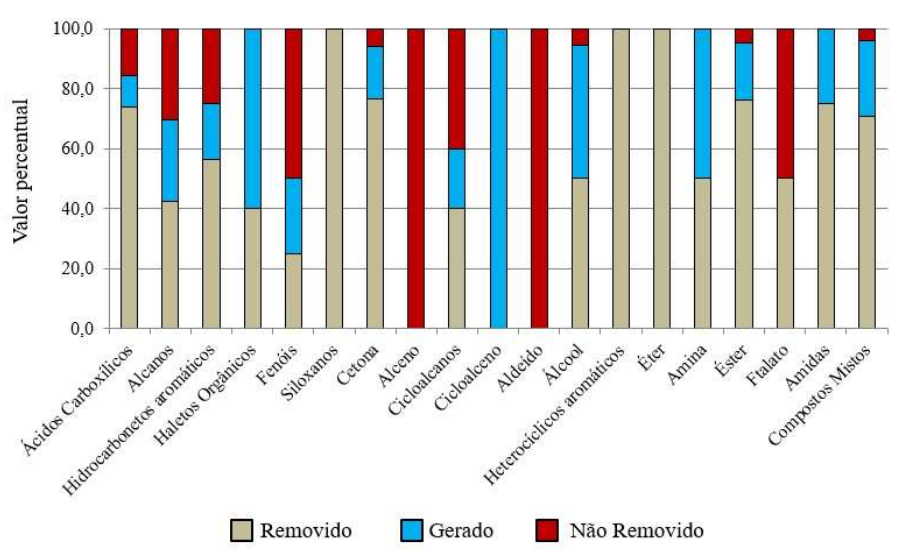

Figura 1: Valor percentual dos compostos orgânicos removidos, gerados e não removidos no EF.

É importante salientar ainda, que o tratamento de efluentes que possuam alto potencial poluidor, toxicidade e características recalcitrantes como o lixiviado é um grande desafio, visto que, mesmo utilizando vários tipos de tratamentos conjugados na maioria das vezes no efluente tratado ainda restam vários compostos orgânicos. Se por um lado a caracterização mais completa do lixiviado permite o melhor entendimento de suas características, por outro lado mostra a necessidade de novos estudos na busca de tratamentos mais eficientes que geralmente são mais complexos e onerosos.

Os dados de algumas pesquisas evidenciam a dificuldade da remoção de compostos orgânicos no lixiviado de aterro sanitário, Reis et al. (2017) utilizaram em sua pesquisa lixiviado proveniente do aterro sanitário de Macaúbas localizado em município de Sabará-MG, duas rotas distintas foram utilizadas no tratamento do lixiviado, Biorreator de Membrana tradicional (BRMb) e Biorreator de Membrana inoculado com a levedura (BRMI) Saccharomyces cerevisiae, seguido de nanofiltração, as duas rotas eram precedidas de uma etapa de air stripping. No lixiviado bruto foram identificados aproximadamente 100 compostos orgânicos. Com relação as rotas utilizadas no tratamento do lixiviado o BRMI alcançou melhor eficiência na remoção de compostos orgânicos (80\%).

A utilização da nanofiltração como polimento, elevou a remoção de compostos orgânicos nas rotas BRMb e BRMI para aproximadamente $90 \%$ e $92 \%$ nas duas rotas. Assim como ocorreu com o LIN do município de Puxinanã-PB, a maior parte dos compostos foram removidos, mas houve a formação de vários outros compostos. Dos compostos que persistiram a nanofiltração está o benzaldeído, o mesmo composto permaneceu no EF.

He et al. (2015) pesquisaram a eficácia do processo Fenton como pós tratamento de um lixiviado proveniente do aterro na província de Zhejiang na China. O lixiviado foi previamente tratado biologicamente e posteriormente tratado por sistemas de membranas, ultra e nanofiltração e o concentrado deste processo foi coletado e encaminhado para ser tratado por processo Fenton. O estudo se concentrou na avaliação de 
remoção de três poluentes prioritários, o metil benzeno, o etil benzeno e o cloro benzeno encontrados no lixiviados com nas seguintes concentrações (mg/L), 3,5, 0,44 e 0,73 respectivamente. O processo Fenton se mostrou eficiente na remoção de $50 \%$ do metil benzeno, $46,3 \%$ do etil benzeno e $21,4 \%$ do cloro benzeno. Destes compostos identificados e quantificados no aterro da China, apenas o etil benzeno foi encontrado no EF do lixiviado do aterro de Puxinanã.

\section{CONCLUSÕES}

Os resultados obtidos evidenciam que o processo Fenton apresenta características operacionais promissoras no tratamento de lixiviados de aterro sanitário, principalmente àqueles que apresentam baixa relação $\mathrm{DBO}_{5} / \mathrm{DQO}$, como o lixiviado do aterro do município de Puxinanã-PB. No entanto, sua eficiência é limitada, não alcançando os limites legais para lançamento em corpos hídricos.

Após otimização dos parâmetros operacionais, foi alcançado remoção $87 \%$ de DQO, 74,4\% de COD e $91,3 \%$ de cor verdadeira. Houve remoção de $85 \%$ de nitrogênio amoniacal e foi observado ainda aumento na razão $\mathrm{DBO}_{5} / \mathrm{DQO}$ de 0,33 para 0,77 . Verificou-se pequena redução na concentração de sólidos totais e redução de 70\% dos sólidos voláteis e redução da relação SV/ST de 0,29 para 0,10.

Em relação aos compostos orgânicos, foram identificados 154 no LIN e 79 no EF. A eficiência do processo Fenton na remoção dos compostos orgânicos foi de $80 \%$, dos compostos orgânicos que permaneceram no EF, alguns são reconhecidamente tóxicos. No EF foram identificados 50 novos subprodutos.

Dos compostos classificados como desregulados endócrinos foram identificados no LIN o Di-isoctil ftalato, o Di-(2-propilpentil) éster ftálico e o 4,4-isopropilideno di-fenol mais conhecido com Bisfenol A, destes, o último permaneceu no EF.

No EF foram identificados vários compostos reconhecidamente tóxicos, são eles, bisfenol A, 2,4-diterc-butilfenol (2,4-DTBF), bezaldeído, cloro benzeno, fenol, vários compostos aromáticos, peroxido de hidrogênio residual e alta concentração de ferro.

A elaboração desta pesquisa evidenciou a necessidade de mudança na legislação vigente no sentido de ampliar o monitoramento de algumas substâncias tóxicas presente no LIN.

\section{REFERÊNCIAS}

AKKAYAA, G. K.; BILGILIB, M. S.. Evaluating the performance of an electro-membrane bioreactor in treatment of young leachate. Journal of Environmental Chemical Engineering, v.8, n.4, p.1-10, 2020. DOI:

https://doi.org/10.1016/j.jece.2020.104017

ANFRUNS, A.; GABARRÓ, J.; GONZALEZ-OLMOS, R.; PUIG, S.; BALAGUER, M. D.; COLPRIM, J.. Coupling anammox and advanced oxidation-based technologies for mature landfill leachate treatment. Journal of Hazardous Materials, v.258259, p.27-34, 2013. DOI:

https://doi.org/10.1016/j.jhazmat.2013.04.027

APHA; AWWA; WEF. American Public Health Association; American Water Works Association; World Economic Forum. Standard Methods for the Examination of Water and
Wastewater. APHA, 2012.

BABUPONNUSAMI, A.; MUTHUKUMAR, K.. A review on Fenton and improvements to the Fenton process for wastewater treatment. J. Environ. Chem. Eng., v.2, p.557572, 2014. DOI: https://doi.org/10.1016/j.jece.2013.10.011

BAUTISTA, P.; MOHEDANO, A. F.; CASAS, J. A.; ZAZO, J. A.; RODRIGUEZ, J. J.. Review: An overview of the application of Fenton oxidation to industrial wastewaters treatment. Journal of Chemical Technology and Biotechnology, v.83, p.1323-1338, 2008. DOI: https://doi.org/10.10002/jctb.1988

BILA, D. M.; DEZOTTI, M.. Desreguladores endócrinos no meio ambiente: efeitos e consequências. Química Nova, 
v.30, n.3, p.651-666, 2007. DOI:

https://doi.org/10.1590/S0100-40422007000300027

BEGUM, S.; ARELLI, V.; ANUPOJU, G. R.; SRIDHAR, S.; BHARGAVA, S. K.; ESHTIAGHI, N.. Optimization of feed and extractant concentration for the liquid-liquid extraction of volatile fatty acids from synthetic solution and landfill leachate. Journal of Industrial and Engineering Chemistry, v.90, p.190-202, 2020. DOI:

https://doi.org/10.1016/i.jiec.2020.07.011

BREITKREITZ, M. C.; SOUZA, A. M.; POPP, R. J.. Experimento didático de quimiometria para planejamento de experimentos: avaliação das condições experimentais na determinação espectrofotométrica de ferro II com $\alpha$ fenantrolina, parte III. Química Nova, v.37, n.3, p.564-573, 2014. DOI: https://doi.org/10.5935/0100-4042.20140092

CASSANO, D.; ZAPATA, A.; BRUNETTI, G.; DEL MORO, G.; DI LACINI, C.; OLLER, I.; MALATO, S.. Comparison of several combined/integrated biological-AOPs setups for the treatment of municipal landfill leachate: Minimization of operating costs and effluent toxicity. Chemical Engineering Journal, v.172, n.1, p.205-257, 2011. DOI: https://doi.org/10.1016/j.cej.2011.05.098

CHENG, S. Y.; SHOW, B. P.; JUAN, J. C.; CHANG, J. D. E. F. G.; LAU, B. F.; LAI, S. H.; NG, E. P.; YIAN, H. C.; LING, T. C.. Landfill leachate wastewater treatment to facilitate resource recovery by a coagulation-flocculation process via hydrogen bond. Chemosphere, v.262, p.1-9, 2020. DOI: https://doi.org/10.1016/j.chemosphere.2020.127829

CHRISTENSEN, T. H.; KJELDSEN, P.; BJERG, P. L.; JENSEN, D. L.; CHRISTENSEN, J. B.; BAUN, A.; ALBRECHTSEN, H. J.; HERON, G.. Biogeochemistry of landfill leachate plumes. Applied Geochemistry, v.16, p.659-718, 2001. DOI: https://doi.org/10.1016/S0883-2927(00)00082-2

DANTAS, E. R. B.; SILVA, E. J.; LOPES, W. S.; NASCIMENTO, M. R.; LEITE, V. D.; SOUSA, J. T.. Fenton treatment of sanitary landfill leachate: optimization of operational parameters, characterization of sludge and toxicology. Environmental Technology, v.41, p.1-34, 2019. DOI: https://doi.org/10.1080/09593330.2019.1576773

DU, Y.; ZHOU, M.; LEI, L.. Role of the intermediates in the degradation of phenolic compounds by Fenton-like process. Journal of Hazardous Materials, v.136, p.859-865, 2006. DOI: https://doi.org/10.1016/i.jhazmat.2006.01.022

DUBOIS, M.; GILLES, K. A.; HAMILTON, J. K.; REBERS, P. A.; SMITH, F.. Colorimetric method for determination of sugars and related substance. Analycal Chemistry, v.28, p.350-356, 1956. DOI: https://doi.org/10.1021/ac60111a017

FENTON, H. J. H.. Oxidation of tartaric acid in presence of iron. Journal of the Chemical Society, Transactions, v.65, p.899-910, 1894. DOI:

https://doi.org/10.1039/СТ8946500899

FERNANDES, A.; PACHECO, M. J.; CIRÍACO, L.; LOPES, A.. Review on the electrochemical processes for the treatment of sanitary landfill leachates: Present and future. Applied Catalysis B: Environmental, v.176-177, p.183-200, 2015. DOI: https://doi.org/10.1016/j.apcatb.2015.03.052
FLOLUND, B.; PALMGREN, R.; KEIDING, K.; NIELSEN, P. H.. Exction os extracelular polymers from activeted sludge using cátion exchange resin. Water Research, v.30, n.8, p.17491758,1995

GAUTAM, P.; KUMAR, S.; LOKHANDWALA, S.. Advanced oxidation processes for treatment of leachate from hazardous waste landfill: A critical review. Journal of Cleaner Production, v.237, p.212-222, 2019. DOI: https://doi.org/10.1016/i.jclepro.2019.117639

GÖDE, J. N.; SOUZA, D. H.; TREVISAN, V.; SKORONSKI, E.. Application of the Fenton and Fenton-like processes in the landfill leachate tertiary treatment. Journal of Environmental Chemical Engineering, v.7, p.1-8, 2019. DOI: https://doi.org/10.1016/j.jece.2019.103352

HE, R.; TIAN, B.; ZHANG, Q.; ZHANG, H.. Effect of Fenton oxidation on biodegradability, biotoxicity and dissolved organic matter distribution of concentrated landfill leachate derived from a membrane process. Waste Management, v.38, p.232-239, 2015. DOI:

https://doi.org/10.1016/j.wasman.2015.01.006

HUO, S.; XI, B.; YU, H.; HE, L.; FAN, S.; LIU, H.. Characteristics of dissolved organic matter (DOM) in leachate with different landfill ages. Journal of Environmental Sciences, v.20, n.4, p.492-498, 2008. DOI: https://doi.org/10.1016/S10010742(08)62085-9

HYBSKÁ, H.; LOBOTKOVÁ, M.; VANEKA, M.; SALVA, J.; KNAPCOVÁ, I.; VEVERKOVÁ. D.. Biomonitoring and its in the assessment of the quality of wastewater treatment process. Environmental Nanotechnology, Monitoring \& Management, v.13, p.1-8, 2020. DOI: https://doi.org/10.1016/j.enmm.2020.100292

ISKANDER, M. D. S.; NOVAK, Z. T.; HE, Z.. Reduction of reagent requirements and sludge generation in Fenton's oxidation of landfill leachate by synergistically incorporating forward osmosis and humic acid recovery. Water Research, v.151, p.310-317, 2019. DOI:

https://doi.org/10.1016/j.watres.2018.11.089

KANG, K. H.; SHIN, H. S.; PARK, H.. Characterization of humic substances present in landfill leachates with different landfill ages and its implications. Water Research, v.36, p.40234032, 2002. DOI: https://doi.org/10.1016/S00431354(02)00114-8

KARTHIKEYAN, S.; TITUS, A.; GNANAMANI, A.; MANDAL, A B.; SEKARAN G.. Treatment of textile wastewater by homogeneous and heterogeneous Fenton oxidation processes. Desalination, v.281, p.438-445, 2011. DOI: https://doi.org/10.1016/i.desal.2011.08.019

LANGE, L. C.; ALVE, J. F.; AMARAL, M. C. S. M.; MELO JÚNIOR, W. R.. Tratamento de lixiviado de aterro sanitário por processo oxidativo avançado empregando reagente de Fenton. Revista de engenharia Sanitária e Ambiental, v.11, n.2, p.175-183, 2006. DOI: https://doi.org/10.1590/S1413$\underline{41522006000200011}$

LIU, X.; NOVAK, J. T.; HE, Z.. Synergistically coupling membrane electrochemical reactor with Fenton process to enhance landfill leachate treatment. Chemosphere, v.247, 
p.1-8, 2020. DOI:

https://doi.org/10.1016/i.chemosphere.2020.125954

MÉNDEZ, R. I.; PIETROGIOVANNA, J. A.; SANTOS, B.; SAURI, M. R.; GIÁCOMAN, G.; CASTILLO, E. R.. Determinación de la dosis óptima de reactivo fenton en un tratamiento de lixiviados por Fenton- adsorción. Rev. Int. Contam. Ambient, v.26, n.3, p.211-220, 2010.

MENDONÇA, E.; PICADOA, A.; PAIXÃOA, S. M.; SILVA, L.; CUNHA, M. A.; LEITÃO, S.; MOURA, I.; CORTEZC, C.; BRITO, F.. Ecotoxicity tests in the environmental analysis of wastewater treatment plants: Case study in Portugal. Journal of Hazardous Materials, v.163, p.665-670, 2009. DOI: http://doi.org/10.1016/j.jhazmat.2008.07.012

MORAVIA, W. G.; AMARAL, M. C. S.; LANGE, L. C.. Evaluation of landfill leachate treatment by advanced oxidative process by Fenton's reagent combined with membrane separation system. Waste Management, v.33, p.89-101, 2013. DOI: https://doi.org/10.1016/i.wasman.2012.08.009

MORAVIA, W. G.; LANGE, L. C.; AMARAL, M. C. S.. Avaliação do Processo Oxidativo Avançado pelo reagente Fenton em condições otimizadas no tratamento de lixiviado de aterro sanitário com ênfase em parâmetros coletivos e caracterização do lodo gerado. Química Nova, v.34, n.8, p.1370-1377, 2011. DOI: https://doi.org/10.1590/S0100$\underline{40422011000800014}$

NASCIMENTO FILHO, I.; MUHLEN, C. V.; CARAMÃO, E. B. Estudo de compostos orgânicos em lixiviados de aterros sanitários por EFS e CG/EM. Química Nova, v.24, n.4, p.554556, 2001. DOI: https://doi.org/10.1590/S010040422001000400017

NOGUEIRA, R. F. P.; OLIVEIRA, M.C.; PATERLINI, W. C.. Simple and fast spectrophotometric determination of $\mathrm{H}_{2} \mathrm{O}_{2}$ in photo-Fenton reactions using metavanadate. Talanta, v.66, p.86-91, 2005. DOI:

https://doi.org/10.1016/i.talanta.2004.10.001

NOGUEIRA, R. F. P.; TROVÓ, A. G.; SILVA, R. A.; VILLA, R.D. Fundamentos e aplicações ambientais dos processos Fenton e Foto-Fenton. Química Nova, v.30, n.2, p.400-408, 2007. DOI: https://doi.org/10.1590/S0100-40422007000200030

PAIVA, F. V.; SOUZA, N. C.; VAN HAANDEL, A. C.. Identificação de compostos orgânicos e farmacêuticos em esgoto hospitalar utilizando cromatografia gasosa acoplada a espectrometria de massa. Engenharia Sanitária e Ambiental, v.16, n.1, p.37-44, 2011. DOI: https://doi.org/10.1590/S1413-41522011000100007

PELLENZ, L.; BORBA, F. H.; DAROIT, D. J.; LASSEN, M. F. M.; BARONI, S.; ZORZO, C. F.; GUIMARÃES, R. E.; ESPINOZA, Q. F. R.; SEIBERT, D.. Landfill leachate treatment by a borondoped diamond-based photo-electro-Fenton system integrated with biological oxidation: A toxicity, genotoxicity and by products assessment. Journal of Environmental Management, v.264, p.1-12, 2020. DOI: https://doi.org/10.1016/j.jenvman.2020.110473

PERALTA-ZAMORA, P. G.; KUNZ, A.; MORAES, S. G.; PELEGRINI, R.; MOLEIRO, P. C.; REYES, J.; DURÁN, N.. Degradation of reactive dyes I. A comparative study of ozonation, enzymatic and photochemical processes.

Chemosphere, v.38, p.835-852, 1999. DOI:

https://doi.org/10.1016/S0045-6535(98)00227-6

PLATA, G. B.; ALFANO, O. M.; CASSANO, A. E.

Decomposition of 2-chlorophenol employing goethite as Fenton catalyst. I. Proposal of a feasible, combined reaction scheme of heterogeneous and homogeneous reactions.

Applied Catalysis B Environmental, v.95, p.1-13, 2010. DOI: https://doi.org/10.1016/i.apcatb.2009.12.005

POBLETE, R. PEREZ, N.. Use of sawdust as pretreatment of photo-Fenton process in the depuration of landfill leachate. Journal of Environmental Management, v.253, p.1-8, 2020 DOI: http://doi.org/10.1016/j.jenvman.2019.109697

POHLAND, F. G.; HARPER, S. R.. Critical review and summary of leachate and gasproduction from landfills. Tech Project n. E20 G01, 1985.

RANI, A. D.; NEGIB, S. D.; HUSSAINC, A.; KUMAR, S. Treatment of Urban Municipal Landfill Leachate Utilizing Garbage Enzyme. Bioresource Tchnology, v.297, p.1-23, 2020. DOI: https://doi.org/10.1016/j.biortech.2019.122437

REIS, B. G.; SILVEIRA, A. L.; LEBRON, Y. A. R.; MOREIRA, V. R.; TEIXEIRA, L. P. T.; OKUMA, A. A.; AMARAL, M. C. S.; LANGE, L.. Comprehensive investigation of landfill leachate treatment by integrated fenton/microfiltration and aerobic membrane bioreactor with nanofiltration. Process Safety and Environmental Protection, v.143, p.121-128, 2020. DOI: https://doi.org/10.1016/j.psep.2020.06.037

REIS, B. G.; SILVEIRA, A. L.; TEIXEIRA, L. P. T.; OKUMA, A. A.; LANGE, L. C.; AMARAL, M. C. S.. Organic compounds removal and toxicity reduction of landfill leachate by commercial bakers' yeast and conventional bacteria based membrane bioreactor integrated with nanofiltration. Waste Management, v.70, p.170-180, 2017. DOI: https://doi.org/10.1016/j.wasman.2017.09.030

RENOU, S.; GIVAUDAN, J. G.; POULAIN A, S.; DIRASSOUYAN, F.; MOULIN, P.. Landfill leachate treatment: Review and opportunity. Journal of Hazardous Materials, v.150, p.468493, 2008. DOI:

https://doi.org/10.1016/j.jhazmat.2007.09.077

RIGOBELLO, E. S.; SCANDELAI, A. P. J.; CORSO, B. L.; TAVARES, C. R. G.. Identificação de compostos orgânicos em lixiviado de aterro sanitário municipal por cromatografia gasosa acoplada a espectrometria de massas. Química Nova, v.38, n.6, p.794-800, 2015. DOI: http://dx.doi.org/10.5935/0100-4042.20150092

ROSA, A. H.; ROCHA, J. C.; FURLAN, M.. Substâncias húmicas de turfa: estudo dos parâmetros que influenciam no processo de extração alcalina. Química Nova, v.23, n.4, p.472-476, 2000. DOI: https://doi.org/10.1590/S0100$\underline{0422000000400008}$

ROUDI, A. M.; CHELLIAPAN, S.; KAMYAB, H.; DIN, M.; FADHIL, M.; KRISHNAN, S.. Removal of COD from landfill leachate by Predication and Evaluation of Multiple Linear Regression (MLR) Model and Fenton process. Egyptian Journal of Chemistry, v.62, n.7, p.1207-1618, 2019. DOI: http://doi.org/10.21608/EJCHEM.2018.6429.1543 
ROUDI, A. M.; KAMYAB, H.; CHELLIAPAN, S.; ASHOKKUMAR, V.; KUMAR, A.; YADAV, K. K.; GUPTA, N.. Application of response surface method for Total organic carbon reduction in leachate treatment using Fenton process. Environmental Technology \& Innovation, v.19, p.1-26, 2020. DOI: https://doi.org/10.1016/j.eti.2020.101009

SABOUR, M. R.; AMIRI, A.. Comparative study of ANN and RSM for simultaneous optimization of multiple targets in Fenton treatment of landfill leachate. Waste Management, v.65, p.54-62, 2017. DOI: 10.1016/j.wasman.2017.03.048

SALEEM, M.; MASUTA, E.; SPAGNIB, A.; LAVAGNOLO, M. C.. Exploring dynamic membrane as an alternative for conventional membrane for the treatment of old landfill leachate. Journal of Environmental Management, v.246, p.658-667, 2019. DOI: https://doi.org/10.1016/i.jenvman.2019.06.025

SAUVE, G.; ACKER, K.. The environmental impacts of municipal solid waste landfills in Europe: A life cycle assessment of proper reference cases to support decisionmaking. Journal of Environmental Management, v.261, p.112, 2020. DOI:

https://doi.org/10.1016/j.jenvman.2020.110216.

SILVA, F. L. N.; SANTOS JUNIOR, J. R.; MOITA NETO, J. M.; SILVA, R. L. G. N. P.; FLUMIGNAN, D. L.; OLIVEIRA, J. E.. Determinação de benzeno, tolueno, etilbenzeno e xilenos em gasolina comercializada nos postos do estado do Piauí. Química Nova, v.32, n.1, p.56-60, 2009. DOI: http://dx.doi.org/10.1590/S0100-40422009000100011

SIMÃO, F. C. P.; GRAVATO, C.; MACHADO, A. L.; SOARES, A M. V. M.; PESTANA, J. L. T.. Toxicity of different polycyclic aromatic hydrocarbons (PAHs) to the freshwater planarian Girardia tigrina. Environmental Pollution, v.266, p.1-12, 2020. DOI: https://doi.org/10.1016/i.envpol.2020.115185

SUN, H.; PENG, Y.; SHI, X.. Advanced treatment of landfill leachate using anaerobic-aerobic process: Organic removal by simultaneous denitritation and methanogenesis and nitrogen removal via nitrite. Bioresource Technology, v.177, p.337-345, 2015. DOI: https://doi.org/10.1016/j.biortech.2014.10.152

TIGINI, V.; PRIGIONE, V.; VARESE, G. C.. Mycological and ecotoxicological characterisation of landfill leachate before and after traditional treatments. Science of the Total Environment, v.487, p.335-341, 2014. DOI: https://doi.org/10.1016/j.scitotenv.2014.04.026

TURKI, N.; BELHAJ, D.; JAABIRI, I.; AYADI, H.; KALLEL, M.; BOUZID, J.. Determination of organic compounds in landfill leachates treated by coagulation-flocculation and fentonadsorption. IOSRJ Journal of Environmental Science, Toxicology and Food Technology, v.7, p.18-25, 2013. DOI: http://doi.org/10.9790/2402-0731825
UMAMAHESWARI, J.; BHARATHKUMAR, T.; SHANTHAKUMAR, S.; GOTHANDAM, K. M.. A Feasibility Study on Optimization of Combined Advanced Oxidation Processes for Municipal Solid Waste Leachate Treatment. Process Safety and Environmental Protection, v.143, p.212221, 2020. DOI: https://doi.org/10.1016/j.psep.2020.06.040

WANG, D.; TANG, Y.; LONG, G.; HIGGITT, D.; HE, J.; ROBINSON, D.. Future improvements on performance of an EU landfill directive driven municipal solid waste management for a city in England. Waste Management, v.102, p.452-463, 2020. DOI: https://doi.org/10.1016/j.wasman.2019.11.009

WEI, G.; LI, D.; ZHUO, M.; LIAO, Y.; XIE, Z.; GUO, T.; LI, J.; ZHANG, S.; LIANG, Z.. Organophosphorus flame retardants and plasticizers: Sources, occurrence, toxicity and human exposure. Environmental Pollution, v.196, p.29-46, 2015. DOI: https://doi.org/10.1016/j.envpol.2014.09.012

WU, S.; ZOU, S.; LIANG, G.; QIAN, G.; HE, Z.. Enhancing recovery of magnesium as struvite from landfill leachate by pretreatment of calcium with simultaneous reduction of liquid volume via forward osmosis. Science of the Total Environment, v.610-611, p.137-146, 2018. DOI: https://doi.org/10.1016/j.scitotenv.2017.08.038

XIANGLI, L.; LI, L.; SHICHUN, Z.; CHONGYU, L.; TIANGANG, L. Determination of Bisphenol $A$ in Landfill Leachate by Solid Phase Microextraction with Headspace Derivatization and Gas Chromatography-Mass Spectrophotometry. Chinese Journal of Analytical Chemistry, v.34, n.3, p.325-328, 2006. DOI: https://doi.org/10.1016/S1872-2040(06)60018-2

XIAOLI, C.; YONGXIA, H.; GUIXIANG, L.; XIN, Z.; YOUCAI, Z.. Spectroscopic studies of the effect of aerobic conditions on the chemical characteristics of humic acid in landfill leachate and its implication for the environment. Chemosphere, v.91, p.1058-1063, 2013. DOI: http://doi.org/10.1016/j.chemosphere.2013.01.052

XU, Q.; SIRACUSA, G.; BECARELLI, S.; YUAN, Q.. COD removal from biologically stabilized landfill leachate using Advanced Oxidation Processes (AOPs). Process Safety and Environmental Protection, v.120, p.278-285, 2018. DOI: https://doi.org/10.1016/j.psep.2018.09.014

YUAN, Q.; JIA, H.; POVEDA, M.. Study on the effect of landfill leachate on nutrientremoval from municipal waterwater. Journal Environmental Sci., v.43, p.153-158, 2016. DOI: https://doi.org/10.1016/j.jes.2015.10.023

ZHANG, M. H.; HUI-DONG, H.; ZHAO, L.; WANG, D. X.; MENG, D.. A review on Fenton process for organic wastewater treatment based on optimization perspective. Science of the Total Environment, v.670, p.110-121, 2019. DOI: https://doi.org/10.1016/j.scitotenv.2019.03.180

A CBPC - Companhia Brasileira de Produção Científica (CNPJ: 11.221.422/0001-03) detém os direitos materiais desta publicação. Os direitos referem-se à publicação do trabalho em qualquer parte do mundo, incluindo os direitos às renovaç̃es, expansões e disseminaçoses da contribuiç̃o, bem como outros direitos subsidiários. Todos os trabalhos publicados eletronicamente poderão (astes preservam os direitos autorais, mas não têm permissão para a publicação da contribuição em outro meio, impresso ou digital, em português ou em tradução. 